\author{
P. J. Scarpace $\cdot$ M. Matheny $\cdot$ N. Tümer $\cdot$ K. Y. Cheng $\cdot$ \\ Y. Zhang
}

\title{
Leptin resistance exacerbates diet-induced obesity and is associated with diminished maximal leptin signalling capacity in rats
}

Received: 2 December 2004 / Accepted: 12 February 2005 / Published online: 30 April 2005

(C) Springer-Verlag 2005

\begin{abstract}
Aims/hypothesis: Leptin resistance is generally considered a consequence of obesity. We postulated that leptin resistance is associated with diminished hypothalamic leptin signalling capacity and that leptin resistance is causal to obesity. We assessed maximal leptin-mediated binding of the transcription factor signal transducer and activator of transcription 3 (STAT3), and the response to high-fat feeding in lean leptin-resistant rats. Materials and methods: Recombinant adeno-associated virus encoding rat leptin cDNA (rAAV-leptin) or control vector were administered by intracerebroventricular injection to lean F344 $\times$ BN rats for up to 150 days, and food consumption, body weight, serum leptin and glucose tolerance were measured. Leptin-mediated hypothalamic transcription factor binding was assessed at day 150 following an intracerebroventricular injection of $2 \mu \mathrm{g}$ leptin. Rats pretreated with either control or rAAV-leptin vector for 94 days were given a high-fat diet, and energy intake, body weight gain and adiposity were examined. Results: The rAAV-leptin-treated rats initially responded to leptin gene delivery then became leptin-resistant. They displayed persistent submaximal hypothalamic leptin signalling and enhanced insulin sensitivity, yet maximal hypothalamic signalling capacity was decreased by more than $50 \%$. On a high-fat diet, the leptinresistant rats consumed more energy, gained more weight and accumulated greater visceral fat mass than controls. Conclusions/interpretation: The maximal hypothalamic leptin signalling capacity was diminished in leptin-resistant
\end{abstract}

\footnotetext{
P. J. Scarpace $(\bowtie) \cdot M$. Matheny $\cdot$ N. Tümer $\cdot$

K. Y. Cheng $\cdot$ Y. Zhang

Department of Pharmacology and Therapeutics, University of Florida College of Medicine,

Gainesville, FL, USA

e-mail: scarpace@ufl.edu

Tel.: +1-352-3928435

Fax: +1-352-3929696

P. J. Scarpace $\cdot$ N. Tümer $\cdot$ Y. Zhang

Geriatric Research, Education and Clinical Center, Department of Veterans Affairs Medical Center, Gainesville, FL, USA
}

rats receiving central rAAV-leptin gene therapy. Moreover, this leptin-invoked leptin resistance perturbs the regulation of energy homeostasis in response to high fat exposure, producing augmented energy consumption. This, coupled with potential hypersensitivity to insulin, creates a milieu favouring fat deposition. Our data suggest that leptin resistance is both a consequence and cause of obesity.

Keywords Central leptin gene therapy · Diet-induced obesity $\cdot$ F344 $\times$ BN rats $\cdot$ High-fat diet $\cdot$ Leptin-induced leptin resistance $\cdot$ Leptin resistance $\cdot$ Maximal leptin signalling capacity $\cdot$ P-STAT3 transcription factor binding . STAT3 phosphorylation

Abbreviations ACSF: artificial cerebrospinal fluid . i.c.v.: intracerebroventricular - P-STAT3: phosphorylated STAT3 - PMSF: phenylmethylsulphonyl fluoride - PWAT: perirenal white adipose tissue $\cdot$ rAAV-leptin: recombinant adeno-associated virus encoding rat leptin cDNA - RTWAT: retroperitoneal white adipose tissue - SOCS3: suppressor of cytokine signalling- $3 \cdot$ STAT3: signal transducer and activator of transcription 3

\section{Introduction}

Most obese humans and rodents have elevated serum leptin and impaired physiological responses to exogenously administered leptin, and are hence considered leptin-resistant [1-5]. In contrast, in lean rodents leptin evokes potent anorectic and thermogenic responses and produces marked lipopenia and weight reduction [6-8]. For example, chronic hypothalamic overexpression of leptin by recombinant adeno-associated viral-mediated central leptin (rAAV-leptin) delivery in young lean $\mathrm{F} 344 \times \mathrm{BN}$ rats reduces food intake and increases oxygen consumption, resulting in a dramatic loss of fat mass $[9,10]$. However, these leptin responses wane over time despite continued leptin transgene expression, and the decreased adiposity and weight loss are reversed, indicating the development of leptin resistance [10]. Moreover, a central infusion of a pharma- 
cological dose of leptin fails to initiate any physiological response in these rAAV-leptin-treated young rats, confirming their leptin-resistant state [10]. This leptin resistance occurs even in the absence of obesity [10].

Paradoxically, irrespective of the development of this leptin resistance, the continued central overexpression of leptin induces persistent hypothalamic leptin signalling, as evidenced by elevated phosphorylation of signal transducer and activator of transcription 3 (P-STAT3) [9, 11]. It follows from this that leptin resistance can be presumed to be downstream of STAT3 phosphorylation or resident in a parallel signalling cascade. On the other hand, the level of persistently elevated P-STAT3 in leptin-induced leptin-resistant rats may be only submaximal. This is indeed the case in our observation with diet-induced obese rats [12]. We detected a two-fold increase in basal levels of P-STAT3 in the leptin-resistant diet-induced obese animals relative to the lean control rats. But the incremental increase and maximal level of P-STAT3 is diminished in the obese versus lean counterparts in response to an exogenous leptin challenge [12]. It is quite possible that the prolonged exposure to elevated central leptin activates steadily a submaximal level of leptin signalling yet causes a reduction in maximum leptin signal transduction capacity. One goal of the present study is to determine whether the persistently elevated leptin signalling associated with leptin-induced leptin resistance is truly submaximal by assessing maximal leptinmediated P-STAT3 transcription factor binding.

Leptin resistance is common to most obese rodents, whether associated with genetic, diet-induced or age-related obesity. However, whether leptin resistance itself promotes obesity remains unclear. The second goal of the present study was to examine if leptin-induced leptin resistance has functional consequences in young rats. Specifically, we sought to examine if lean rats with leptin-induced leptin resistance are more susceptible to diet-induced obesity. To these ends, we induced hypothalamic leptin resistance in lean rats through administration of rAAV-leptin or control vector encoding green fluorescent protein by intracerebroventricular (i.c.v.) injection. Following the development of leptin resistance, the rats were challenged either with exogtenous leptin to assess maximal signalling capacity or with a high-fat diet to assess susceptibility to obesity.

\section{Materials and methods}

\section{Experimental animals}

Three-month-old male F344×Brown Norway $($ F344×BN) rats were obtained from Harlan Sprague-Dawley (Indianapolis, IN, USA). Upon arrival, rats were examined and remained in quarantine for 1 week. Animals were cared for in accordance with the principles of the Guide to the Care and Use of Experimental Animals by the Canadian Council on Animal Care. Rats were housed individually with a $12: 12 \mathrm{~h}$ light:dark cycle (07.00-19.00 hours).
Experimental design and diet

This study consisted of two experiments (Fig. 1). In each experiment, there were two groups of rats: those administered control vector and those administered rAAV-leptin by i.c.v. injection. Control and rAAV-leptin-treated rats were allowed access to food and water ad libitum, and food consumption and body weight were recorded daily to weekly. In the first experiment, rats were pretreated with control vector or rAAV-leptin for 150 days and then administered artificial cerebrospinal fluid (ACSF) or $2 \mu \mathrm{g}$ leptin, by i.c.v. injection into the lateral ventricle, for assessment of leptinmediated transcription factor binding. In the second experiment, after 69 days of pretreatment with control vector or rAAV-leptin, rats were tested for glucose tolerance. Ninetyfour days after pretreatment with control vector or rAAVleptin, the control group was divided, with one half continued on standard chow (15\% fat; $3.3 \mathrm{kcal} / \mathrm{g}$ diet 2018; Harlan Teklad, Madison, WI, USA) and the other half, together with the group pretreated with rAAV-leptin, fed a high-fat diet (60\% fat; $5.2 \mathrm{kcal} / \mathrm{g}$ D12492; Research Diets, New Brunswick, NJ, USA) for an additional-18 day period.

rAAV-leptin and acute leptin administration

Preparation of the rAAV vector (pTR- $\beta-\mathrm{ObW}$ ) encoding rat leptin cDNA (a generous gift of Roger Unger, University of Texas, Southwestern Medical Center, Dallas, TX, USA [13]) under the control of a chicken $\beta$-actin promoter linked to cytomegalovirus enhancer has been described previously [11]. Vectors were packaged, purified, concentrated
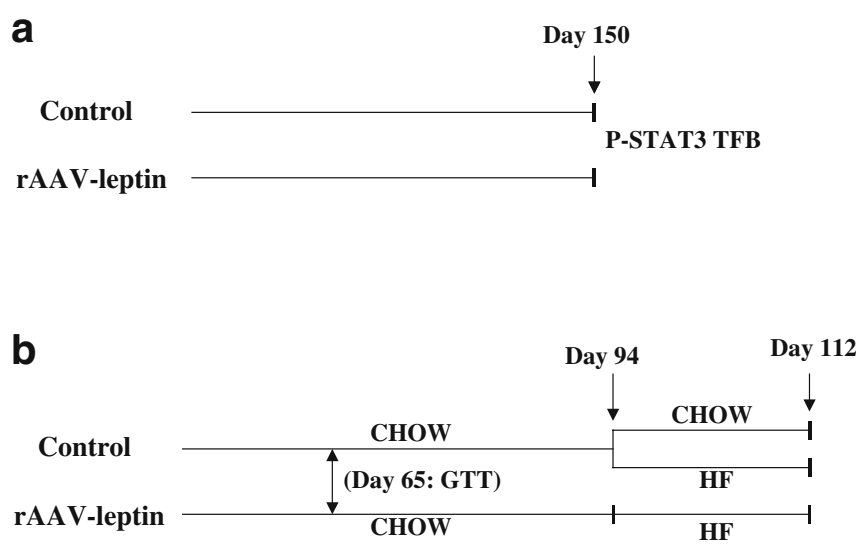

Fig. 1 Experimental schemes. In each experiment, there were two groups of rats, those administered control vector (Control) and those administered rAAV-leptin by i.c.v. injection. In the first experiment (a), rats were pretreated with control vector or rAAV-leptin for 150 days and then administered ACSF or $2 \mu \mathrm{g}$ leptin by i.c.v. injection into the lateral ventricle for assessment of leptin-mediated transcription factor binding $(T F B)$. In the second experiment (b), after 69 days of pretreatment with control vector or rAAV-leptin, rats were tested for glucose tolerance (GTT). At day 94, the control group was divided and one half continued on standard chow (Chow) and the other half of the control group together with the group pretreated with rAAV-leptin were given a high-fat diet $(H F)$ for an additional 18-day period 
and titred as described previously [14]. Rats were administered a single dose of $3.5 \times 10^{9}$ physical particles/rat $\left(6.6 \times 10^{7}\right.$ infectious particles/rat) in $4 \mu \mathrm{l}$ of either control vector or rAAV-leptin by i.c.v. injection into the third cerebral ventricle as follows. Rats were anaesthetised with xylazine, $8 \mathrm{mg} / \mathrm{kg}$ s.c. and $5 \mathrm{~min}$ later $90 \mathrm{mg} / \mathrm{kg}$ ketamine i.p. on the morning of the experiment. After a surgical plane of anaesthesia had been reached, the animal's head was prepared for surgery and the animal was placed into a stereotaxic frame. A small incision $(1.5 \mathrm{~cm})$ was made over the midline of the skull to expose the landmarks of the cranium (bregma and lambda). The coordinates for injection into the third cerebral ventricle were $1.3 \mathrm{~mm}$ anterior to bregma, 9.6 $\mathrm{mm}$ ventral from the skull surface, at an angle of $20^{\circ}$ anterior to posterior. A small hole was drilled through the skull and a 23-gauge stainless steel guide cannula was inserted, followed by an injection cannula. A 3- $\mu$ l volume was delivered using a $10-\mu$ l syringe [9].
For acute administration of leptin into the lateral ventricle, rats were fasted overnight and prepared for surgery as above. The coordinates for injection into the lateral ventricle were $1.3 \mathrm{~mm}$ posterior to bregma, $1.9 \mathrm{~mm}$ lateral to the midline and $3.7 \mathrm{~mm}$ ventral from the skull surface. A small hole was drilled through the skull and a 23-gauge stainless steel guide cannula was inserted, followed by an injection cannula. Using a $50-\mu 1$ syringe, a $5-\mu 1$ volume containing $2 \mu \mathrm{g}$ of murine leptin or ACSF was delivered. Rats were killed $1 \mathrm{~h}$ after injections and remained anaesthetised throughout this period.

\section{Intraperitoneal glucose tolerance test}

An intraperitoneal glucose tolerance test was performed at day 69 after vector administration. Rats were fasted overnight and injected intraperitoneally with glucose $(2 \mathrm{~g} / \mathrm{kg}$
Fig. 2 Daily food consumption (a) and body mass (b) in rats following administration of control vector (open circles) or rAAV-leptin (closed circles). The rAAV-leptin and control vectors were administered at day 0 . Note that between day 75 and day 150 food intake of rAAV-control and rAAV-leptin-treated rats was the same, and is not shown on the graph. Values represent the mean \pm SE of six control and 12 rAAVleptin-treated rats. Food consumption differed between control and rAAV-leptin-treated rats between days 4 and day 46 $(p<0.0001$ by ANOVA with repeated measures). Body mass of the rAAV-leptin-treated rats was significantly different from that of control rats from day 15 until day $75(p<0.0001$ by ANOVA with repeated measures)
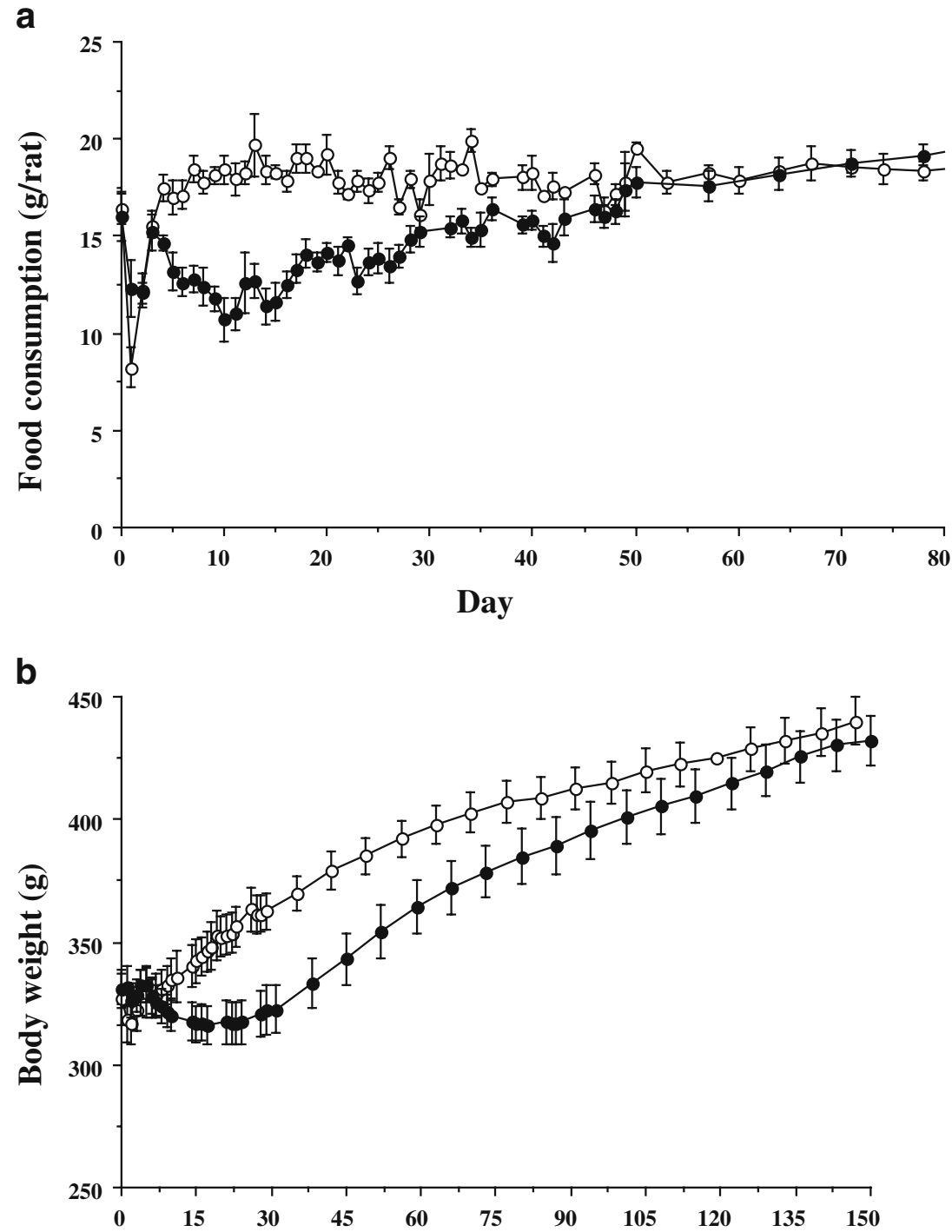

Day 
body weight) at 10.00 hours. Blood was removed from the tail vein immediately before glucose injection and 15, 30, 60 and $120 \mathrm{~min}$ after injection. Blood glucose was measured with a One Touch SureStrep glucose meter (LifeScan, Milpitas, CA, USA). Plasma insulin concentrations were measured with a rat insulin ELISA kit (Linco Research, St Charles, MO, USA).

Tissue harvesting and preparation

Rats were killed by cervical dislocation under $150 \mathrm{mg} / \mathrm{kg}$ pentobarbital anaesthesia. Blood samples were collected by heart puncture, and serum was harvested by 10 min of centrifugation in serum separator tubes. The circulatory system was perfused with $20 \mathrm{ml}$ of cold saline, and perirenal and retroperitoneal white adipose tissues (PWAT and RTWAT, respectively) and the hypothalamus were excised. The hypothalamus was removed by making an incision medial to the piriform lobes, caudal to the optic chiasm and anterior to the cerebral crus to a depth of 2-3 mm. Protein concentrations were determined using the DC protein assay kit (BioRad, Hercules, CA, USA).

\section{Preparation of nuclear protein extracts}

Nuclear protein extracts were prepared immediately following removal of the hypothalamus as in our previous study [15]. At the time the animals were killed, hypothalami were homogenised with a glass pestle in $1 \mathrm{ml}$ of $10 \mathrm{mmol} / 1$ HEPES, pH 7.9, $1.5 \mathrm{mmol} / 1 \mathrm{MgCl}_{2}, 10 \mathrm{mmol} / 1$ $\mathrm{KCl}, 0.5 \mathrm{mmol} / 1$ dithiothreitol and $0.2 \mathrm{mmol} / 1$ phenylmethylsulphonyl fluoride (PMSF). Homogenates were incubated for $10 \mathrm{~min}$ on ice to allow complete cell lysis, dounced with a glass pestle, and centrifuged at 3,300 $\times g$ for $15 \mathrm{~min}$. The nuclear pellets were resuspended in $100 \mu \mathrm{l}$ of a low-salt buffer (20 mmol/1 HEPES, pH 7.9, $1.5 \mathrm{mmol} / 1$ $\mathrm{MgCl}_{2}, 20 \mathrm{mmol} / 1 \mathrm{KCl}, 0.2 \mathrm{mmol} / \mathrm{l}$ EDTA, $0.2 \mathrm{mmol} / 1$ PMSF, $0.5 \mathrm{mmol} / 1$ dithiothreitol and $25 \%$ glycerol), followed by slow addition of $100 \mu \mathrm{l}$ of a high-salt buffer (lowsalt buffer with $1.2 \mathrm{~mol} / 1 \mathrm{KCl}$ ). Extracts were incubated on ice for $30 \mathrm{~min}$ on a rocking platform followed by centrifugation at $25,000 \times g$ for $30 \mathrm{~min}$. The supernatant was centrifuged in a Micron 50 ultrafiltration column (Millipore, Bedford, MA, USA) for $15 \mathrm{~min}$ at $4,000 \times g$ at $4^{\circ} \mathrm{C}$ to concentrate and desalt the extract. The remaining supernatant was harvested and protein was determined.

\section{Electrophoretic mobility shift assay}

The electrophoretic mobility shift assay was similar to the method described previously [15]. The SIE M67 oligonucleotide was ${ }^{32} \mathrm{P} 5^{\prime}$ end-labelled with PNK (Promega, Madison, WI, USA). Probe (100 pg, 50,000 c.p.m.) was incubated in $12 \mu \mathrm{l}$ of $4 \%$ Ficoll, $1 \mathrm{mmol} / 1 \mathrm{MgCl}_{2}, 0.1 \mathrm{mmol} / 1$ EGTA, $0.5 \mathrm{mmol} / \mathrm{l}$ dithiothreitol, $40 \mathrm{mmol} / \mathrm{l} \mathrm{KCl}, 20 \mathrm{mmol} / 1$ HEPES, $\mathrm{pH} 7.9,2 \mu \mathrm{g}$ poly(dI-dC), and $15 \mu \mathrm{g}$ of nuclear
Table 1 Serum leptin and insulin levels at various times following rAAV-leptin or control vector

\begin{tabular}{lll} 
Time after treatment with vector (days) \\
\hline Day 69 & Day 84 & Day 150
\end{tabular}

Serum leptin (ng/ml)

$\begin{array}{llll}\text { rAAV-control } & 4.91 \pm 1.08 & 6.45 \pm 1.38 & 6.89 \pm 1.02\end{array}$

$\begin{array}{lll}\text { rAAV-leptin } \quad 2.32 \pm 0.82 & 3.71 \pm 1.10 \quad 7.32 \pm 1.45\end{array}$

Serum insulin $(\mathrm{ng} / \mathrm{ml})$

$\begin{array}{llll}\text { rAAV-control } & 0.79 \pm 0.10 & 3.84 \pm 1.06^{\mathrm{a}} & 2.86 \pm 0.50^{\mathrm{a}} \\ & 0.47 \pm 0.05^{\mathrm{b}} & 1.37 \pm 0.33^{\mathrm{b}} & 4.94 \pm 1.06^{\mathrm{c}}\end{array}$

rAAV-leptin $\quad 0.47 \pm 0.05^{\mathrm{b}} \quad 1.37 \pm 0.33^{\mathrm{b}} \quad 4.94 \pm 1.06^{\mathrm{c}}$

Data are means \pm SE of 6-11 control or rAAV-leptin-treated rats ${ }^{\mathrm{a}} p=0.0001$ for difference over time by one-way ANOVA; $p<0.001$ (day 84) and $p<0.05$ (day 150) for difference from day 69 $\mathrm{b}_{p=0.039}$ (day 69) and 0.011 (day 84) for difference from control by one-way ANOVA

${ }^{\mathrm{c}} p=0.0001$ for difference over time by one-way ANOVA; $p=0.001$ for difference between day 150 and either day 69 or day 84

protein extract for $20 \mathrm{~min}$ at room temperature. Samples were electrophoretically resolved on a $4 \%$ non-denaturing polyacrylamide gel $(80: 1$; acrylamide: bis-acrylamide in $89 \mathrm{mmol} / \mathrm{l}$ Tris- $\mathrm{HCl}, \mathrm{pH} 8.0,89 \mathrm{mmol} / \mathrm{l}$ boric acid, $2 \mathrm{mmol} / \mathrm{l}$ EDTA for $2 \mathrm{~h}$ ). The gels were dried, exposed to a phosphoimaging screen, scanned with a Phosphoimager (Molecular Dynamics, Sunnyvale, CA, USA), and analysed with ImageQuant software (Molecular Dynamics). The respective densities of images are representative of P-STAT3 binding activity and are expressed in arbitrary units.

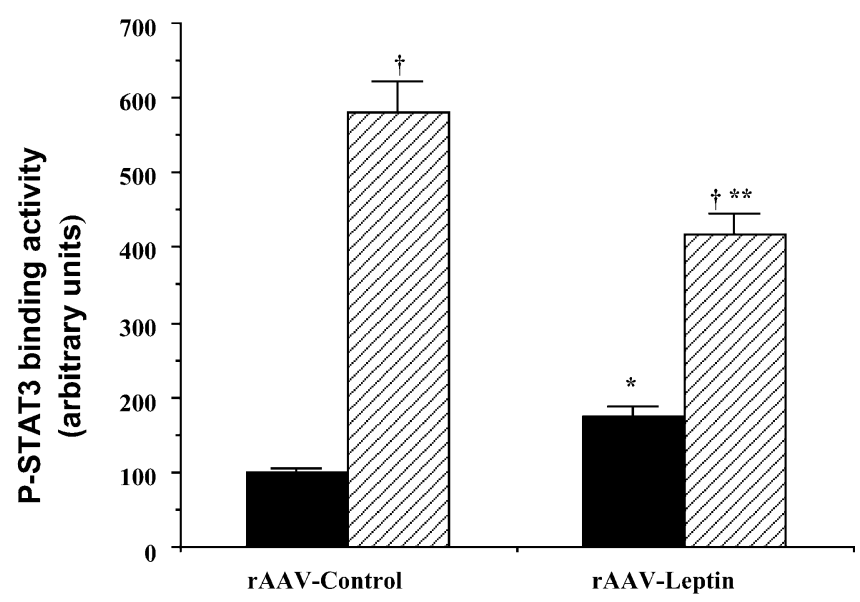

Fig. 3 P-STAT3 transcription factor binding in the hypothalamus following central leptin administration in rats pretreated for 150 days with control vector (solid bars) or rAAV-leptin (hatched bars). Transcription factor binding was assessed $1 \mathrm{~h}$ after administration of leptin ( $2 \mu$ g, i.c.v.). Results are expressed in arbitrary units per microgram of nuclear protein. Levels of transcription factor binding in the control rats administered ACSF were set to 100 and the SE was adjusted proportionally. Values represent the mean $\pm \mathrm{SE}$ of six rats in each group. $p=0.0004$ (pretreatment) and $p=0.0001$ (acute leptin) for difference by two-way ANOVA; ${ }^{*} p=0.0003$ for difference between ACSF-treated rAAV-control and ACSF-treated rAAV-leptin; $* * p=0.023$ for difference between leptin-stimulated rAAV-control and leptin-stimulated rAAV-leptin; ${ }^{\dagger} p=0.0001$ (rAAV-control) and $p=0.003$ (rAAV-leptin) for difference with leptin stimulation compared to respective ACSF controls 
Leptin and insulin and glucose levels

Serum leptin levels were measured using a modified rodent leptin ELISA kit (Crystal Chem, Chicago, IL, USA) or radioimmunoassay kit (Linco Research). Serum insulin levels were measured with a rat insulin RIA kits (Linco Research). Blood glucose was determined with a colorimetric reaction with Trinder, the Sigma Diagnostics Glucose reagent (St Louis, MO, USA).

\section{Statistical analysis}

Data were analysed by one-way or two-way ANOVA. When the main effect was significant, a post-hoc test (TukeyKramer) was applied to determine individual differences between means. A value of $p<0.05$ was considered significant.

\section{Results}

Food consumption and body weight

Following rAAV-leptin administration, food consumption decreased rapidly and became significantly different from that of control rats by day 4 . The reduction in food intake reached a nadir at day 10 , amounting to a $42 \%$ reduction compared with rats administered control vector (Fig. 2a). Starting at day 11, the anorexic response began to wane and food consumption increased gradually, until by day 46 there was no longer a difference between rAAV-leptin-administered and control rats (Fig. 2a).

The rAAV-leptin-treated rats lost body mass initially until day 17 , then gained weight steadily over the remainder of the study. The maximum body mass difference between the rAAV-leptin-treated and control rats was achieved at
Fig. 4 Daily food consumption (a) and body weight gain (b) following a high-fat diet in rats pretreated for 94 days with control vector (open squares) or rAAV-leptin (closed squares) compared with chow-fed rats pretreated with control vector (closed circles). (a) Data are expressed as energy consumption per day, based on $3.3 \mathrm{kcal} / \mathrm{g}$ of chow and $5.2 \mathrm{kcal} / \mathrm{g}$ high-fat diet. Values are mean \pm SE of eight rats per group. In some cases standard error bars are less than the size of the data point. $p<0.001$ for difference in cumulative energy consumption between all pairs (chow control vs high-fat control; chow control vs high-fat rAAV-leptin; and highfat control vs high-fat rAAVleptin by one-way ANOVA). (b) Data express the change in body weight from initiation of the high-fat diet. Values are mean \pm SE of eight rats per group. In some cases standard error bar are smaller than the data point. $p<0.001$ for difference in cumulative weight gain between all pairs (chow control vs high-fat control; chow control vs high-fat rAAV-leptin; and high-fat control vs high-fat rAAV-leptin by one-way ANOVA) a

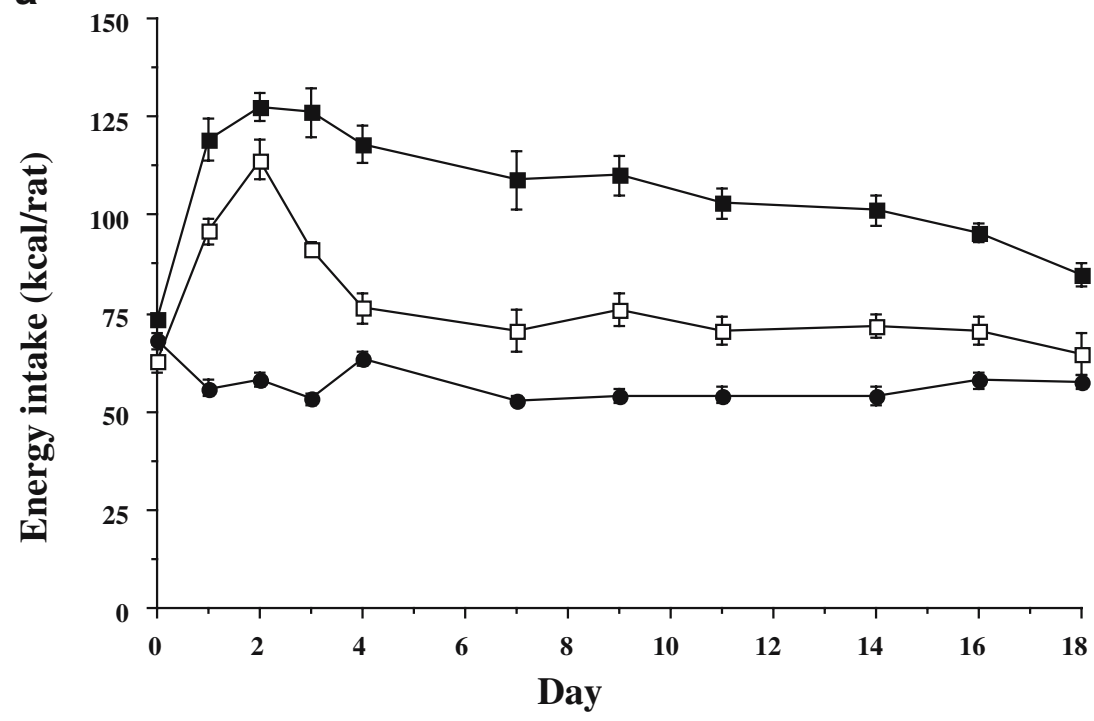

b

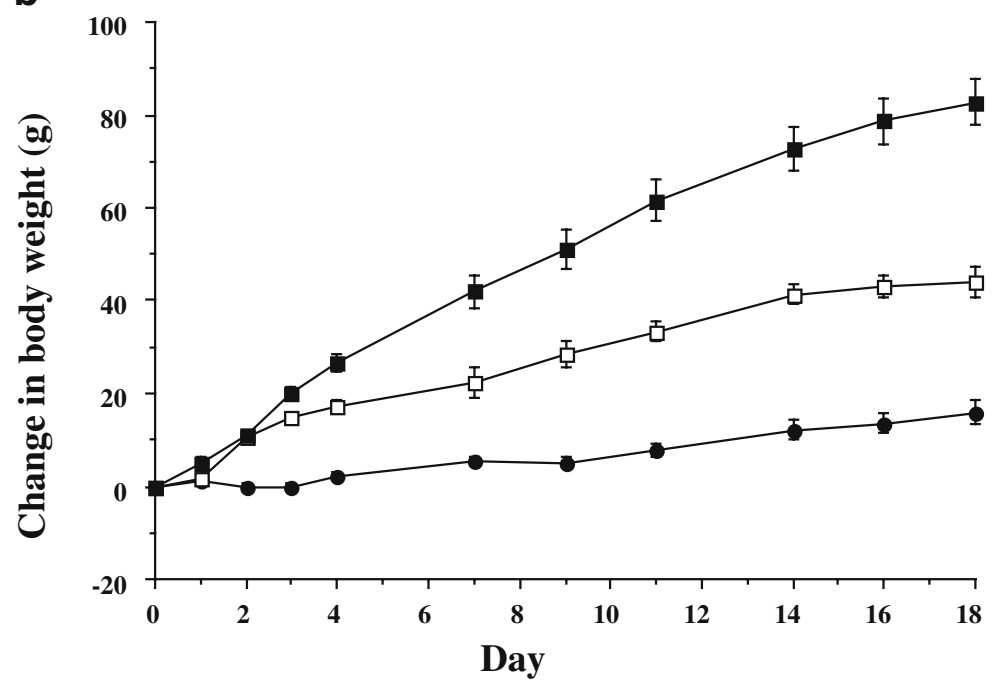


day 30, after which the body masses of the two groups began to converge (Fig. $2 b$ ).

\section{Serum leptin and adiposity levels}

Serum leptin level, one marker of adiposity, was examined at three different times after rAAV-leptin treatment: at day 69 , at which time blood was drawn; at day 84 , when a subset of animals was killed; and at day 150 . By day 69 , food intake was no longer different between the control and rAAV-leptin-treated rats, but body weight was still significantly lower in the latter. At this time, serum leptin was $50 \%$ lower in the rAAV-leptin-treated rats, but this difference was not significant (Table 1). At day 84, during the period when the body weights of the two groups were approaching one another, serum leptin was lower in the leptin gene delivery group, but the difference was not significant. By day 150 , when body weights were nearly identical in the two groups, serum leptin levels were also similar (Table 1). At day 150, adiposity levels, assessed by the sum of the PWAT plus RTWAT, reflected the serum leptin concentration, with no significant difference between the control and rAAV-leptin-treated rats $(7.83 \pm 0.51 \mathrm{~g}$ in rAAV-control vs $7.95 \pm 0.82 \mathrm{~g}$ in the rAAV-leptin-treated group).

The profile of serum insulin was slightly different from that of serum leptin. Serum insulin was significantly lower in rAAV-leptin-treated rats at day 69 and did not change between days 69 and 84, whereas it rose nearly five-fold between days 69 and 84 in the control group (Table 1). In the rats with leptin gene delivery, serum insulin increased more than three-fold between days 84 and 150 , such that the insulin level was no longer significantly different between controls and rAAV-leptin rats at day 150 (Table 1).

Leptin signal transduction in the hypothalamus

Maximal hypothalamic leptin signalling was measured as maximal leptin-induced P-STAT3 transcription factor binding activity using an electrophoretic mobility shift assay, and a dose of central leptin $(2 \mu \mathrm{g})$ previously determined to evoke maximal signalling [15]. Exogenous recombinant mouse leptin or ACSF was introduced into rats that had been pretreated with either rAAV-leptin or control vector for 150 days, as described in Fig. 1, and P-STAT3 transcription factor binding was assessed $1 \mathrm{~h}$ after injection. The basal binding activity was elevated nearly two-fold in the rAAV-leptin-treated rats compared with the control rats (Fig. 3). However, following exogenous leptin stimulation, maximal P-STAT3 transcription factor binding was diminished in the rats pretreated with rAAV-leptin (Fig. 3). In addition, when compared with the respective ACSF-treated rats, those pretreated with control vector and administered exogenous leptin demonstrated an increase of more than five-fold in P-STAT3 transcription factor binding, whereas in rats pretreated with rAAV-leptin and administered exogenous leptin the increase was only two-fold (Fig. 3).
High-fat feeding

To investigate whether this leptin-induced leptin resistance has functional consequences, we challenged the rAAV-leptin-treated rats with a high-fat diet and assessed food intake and body weight. Ninety-four days after pretreatment with rAAV-leptin or control vector, the rats were given a high-fat $\operatorname{diet}(60 \%$ fat) for 18 days and compared with rats pretreated with control vector and maintained on standard chow. Over the 18-day period, the high-fat-fed control rats consumed $43 \%$ more energy (Fig. 4a), gained $175 \%$ more weight (Fig. 4b) and ended with $60 \%$ more visceral fat (Fig. 5) than the chow control. Moreover, the rAAV-leptin high-fatfed rats consumed a $36 \%$ greater amount of energy (Fig. 4a), grew considerably heavier ( $83 \pm 5$ vs $44 \pm 3$ g; Fig. $4 b$ ), and accumulated $26 \%$ more visceral fat (Fig. 5) relative to highfat-fed controls.

\section{Insulin sensitivity}

Prior to the high-fat challenge but 69 days after pretreatment with the rAAV-leptin or control vector, insulin sensitivity was evaluated by an intraperitoneal glucose tolerance test. At this point in the experiment, food consumption was no longer different between the rAAV-leptin and control vector-treated group, and body weight, although converging, was still greater in the control group compared with rAAV-leptin-treated group ( $396 \pm 7$ vs $362 \pm 14 \mathrm{~g}$ ), though not significantly so $(p=0.066)$. Serum leptin levels were also greater in the control group, but again without a significant difference (Table 1). Fasting glucose levels were slightly lower in the rAAV-leptin compared with the control group (69.6 \pm 1.5 vs $64.1 \pm 2.2 \mathrm{mg} / \mathrm{dl}, p=0.045)$, but following the

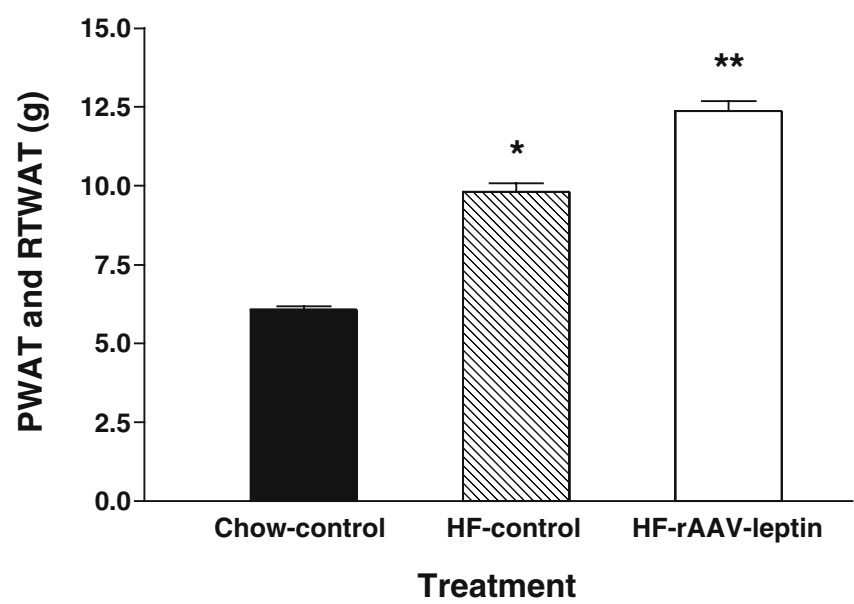

Fig. 5 Adiposity levels following a high-fat diet in rats pretreated for 94 days with control vector (HF-control) or rAAV-leptin (HF-rAAVleptin) compared with chow-fed rats pretreated with control vector (Chow-control). Data represent the sum of the PWAT and RTWAT depots. Values represent the mean \pm SE of eight rats per group. $p<0.0001$ for difference with treatment by one-way ANOVA. ${ }^{*} p<0.001$ (chow control vs high-fat control); ${ }^{* *} p<0.001$ (chow control vs high-fat rAAV-leptin) and $p<0.05$ (high-fat control vs high-fat rAAV-leptin) by post-hoc analysis 
Fig. 6 Blood glucose (a) and serum insulin (b) following glucose loading in rats pretreated for 69 days with control vector (closed circles) or rAAV-leptin (open squares). Values are mean \pm SE of 11 control and eight rAAV-leptin-treated rats. (a) $p=0.045$ for difference in fasting glucose at time zero by one-way ANOVA. There were no significant differences at any time following glucose administration. (b) $p<0.04$ for difference in insulin levels by one-way ANOVA
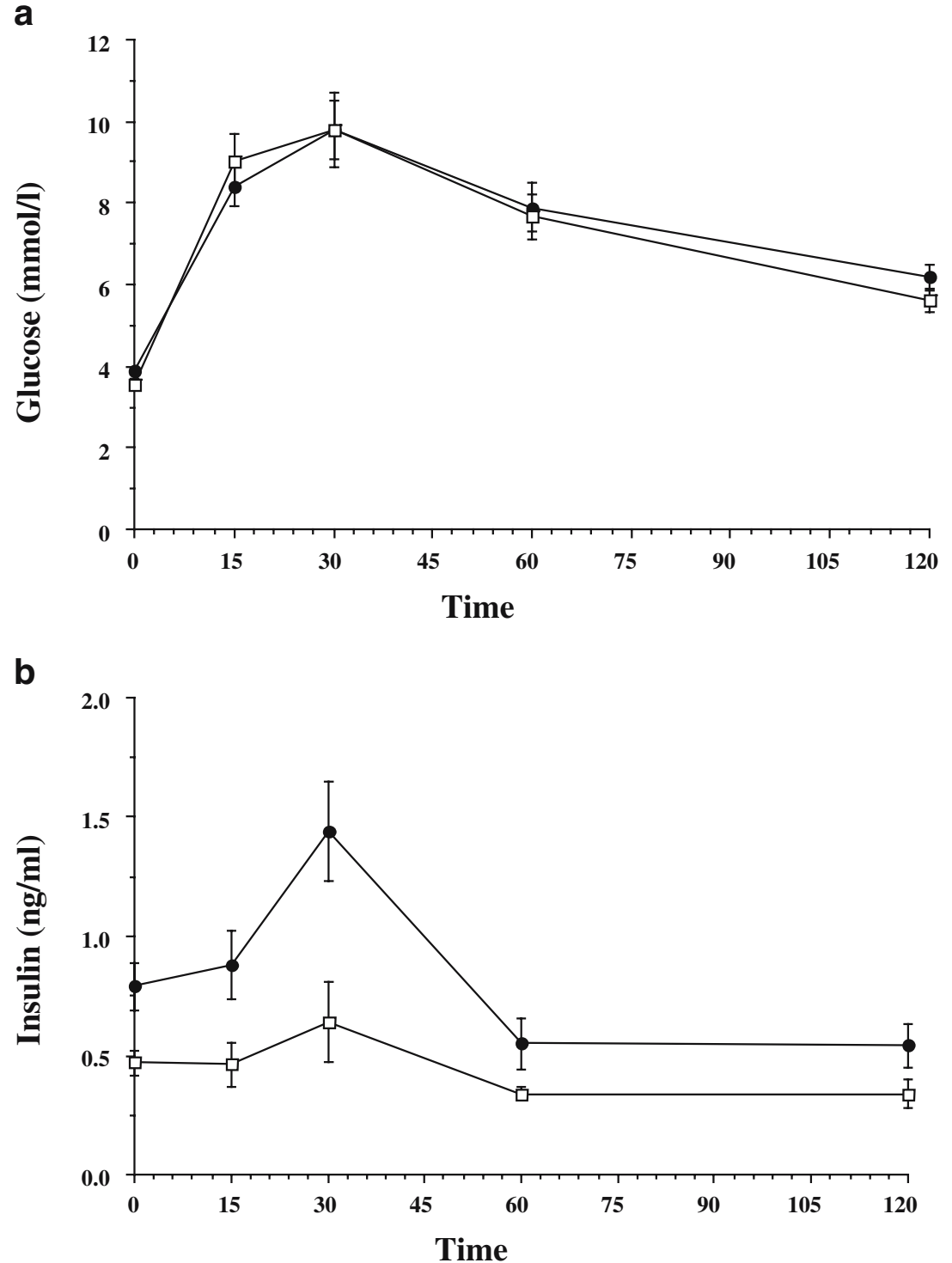

glucose challenge ( $2 \mathrm{~g} / \mathrm{kg}$ body weight) glucose clearance was not different between groups (Fig. 6a). Fasting insulin was lower initially in the rAAV-leptin-treated group compared with the control group $(0.79 \pm 0.10$ vs $0.47 \pm 0.05 \mathrm{ng} / \mathrm{ml}$, $p=0.039$ ), and insulin levels were significantly lower at all time points following the glucose challenge (Fig. 6b).

\section{Discussion}

Diet-induced obesity in rodents is associated with elevated leptin levels [16] and such rats have apparent leptin resistance $[3,4,17]$. Identifying whether leptin resistance contributes to or is simply secondary to obesity is important in understanding the mechanisms underlying obesity. We have previously established a young lean rat model of leptin resistance that avoids the complications of obesity to address this issue [10]. In this model, central overexpression of leptin as a result of long-term rAAV-mediated gene delivery elevates the cerebrospinal fluid leptin level by $75 \%$ [9] and induces leptin resistance characterised by attenuated food and energy responses to the elevated central leptin and lack of these responses to exogenous leptin administration [10].

The present study describes two outcomes of the leptininduced leptin resistance in this model. The first is related to leptin signalling. One puzzling aspect of this leptin resistance is the persistent presence of elevated hypothalamic P-STAT3 in rAAV-leptin-treated rats at approximately twice the level in control rats despite the blunted leptin responses $[9,11]$. However, this two-fold elevation in P-STAT3 is really submaximal compared with the nearly eight-fold stimulation normally produced by an acute dose of central leptin injection [15]. In the present study, in the face of pharmacological stimulation with exogenous leptin, the maximal signalling capacity, as measured by P-STAT3 transcription factor binding to DNA, was reduced in the leptinresistant lean rats compared with the leptin-responsive lean rats. This phenomenon is similar to that described in dietinduced obese and leptin-resistant rodents, where basal leptin signal transduction is elevated [18] yet maximal signalling 
capacity following exogenous central leptin stimulation is diminished [12]. Potentially, the leptin-induced leptin resistance may be linked directly to the impaired maximal leptin signalling capacity.

Several factors may contribute to the decrease in maximal leptin signalling with chronic rAAV-leptin treatment. For instance, suppressor of cytokine signalling-3 (SOCS3) is a leptin-regulated inhibitor of proximal leptin signalling in vivo [19]. SOCS3 inhibits the Janus kinase-mediated phosphorylation of STAT3 and is usually upregulated in response to leptin receptor activation [19]. Our previous data demonstrate that SOCS3 mRNA was elevated either 10 days or 138 days after rAAV-leptin gene delivery [11]. At both of these times, submaximal hypothalamic STAT3 phosphorylation was also elevated [11]. Thus, the increased SOCS3 gene expression did not prevent the sustained submaximal induction of hypothalamic STAT3 phosphorylation by rAAV-leptin. Conceivably, the elevated SOCS3 associated with chronic leptin gene delivery is reducing the maximal leptin signalling, but this remains to be proved. Another potential factor contributing to the decrease in maximal leptin signalling is a reduction in leptin receptors. Chronic exposure to leptin regulates its own receptor expression [20, 21]. In our earlier study, we employed a form of rAAV-leptin containing a tetracycline-responsive promoter. This system allows us to regulate leptin transgene expression via doxycycline in the drinking water [22]. Animals that had their leptin transgene continuously activated for 2 months tended to have reduced hypothalamic leptin receptor expression relative to rats receiving control vector. Furthermore, when the transgene expression was silenced for 1 month, leptin receptor expression increased significantly [22].

The physiological significance of reduced leptin receptors or diminished capacity for leptin signalling with respect to leptin resistance remains in question. Our data indicate that central overexpression of leptin persistently elevates submaximal hypothalamic leptin signalling despite attenuated leptin responses. Presumably, the threshold of leptin signalling necessary to evoke downstream responses may be raised with leptin resistance. That is, greater degrees of activated P-STAT3 transcription factor binding may be necessary to trigger downstream biochemical events. In such a case, any reserve capacity in leptin signalling would be diminished, and physiological responses may be limited by a decrease in leptin receptors or reduced maximal signalling.

Another potential explanation for the dissociation between the persistent submaximally elevated hypothalamic STAT3-related leptin signalling and the impaired leptin physiological responses is that an alternative leptin-mediated signalling pathway accounts for the leptin resistance. The leptin-mediated phosphatidylinositol-3-OHkinase (PI3K) activation pathway may be one candidate [23, 24]. Indeed, impaired activation of PI3K by leptin has been indicated as a novel mechanism of hepatic leptin resistance in diet-induced obesity [25]. However, the role of the central leptin-PI3K pathway in long-term energy homeostasis regulation has not been identified.
The second major finding of this study indicates that central leptin overexpression itself has functional consequences. Treatment by central rAAV-leptin delivery, which leads to both reduced signalling capacity and leptin resistance, exacerbates diet-induced obesity. Leptin resistance is the trademark of diet-induced obesity in rodents $[3,4,17]$ and is generally considered a consequence rather than a cause of obesity. This simple view seems to be at odds with a recent study in transgenic mice overexpressing leptin [26]. The transgenic mice had chronically elevated serum leptin, yet they demonstrated increased weight gain and adiposity when given a high-fat diet, implying a potential interaction between leptin overexpression and high-fat feeding. Because these mice were still hypophagic and leaner than control mice at the time of the high-fat challenge, it was not certain whether these mice were leptin-resistant prior to the high-fat feeding. In contrast, the present study used rAAVleptin-treated rats, a model in which only central leptin is chronically elevated [9], and there is direct evidence of central leptin resistance. Our data indicate that leptin-induced leptin resistance predisposes the animals to diet-induced obesity. It is our contention that leptin resistance is both a consequence and one cause of obesity. An increase in obesity promotes leptin resistance, which in turn exacerbates the obesity, leading to an escalating cycle of increasing obesity. Moreover, our data suggest that one factor contributing to the exacerbated weight gain of the rAAVleptin-treated rats on high-fat feeding is impaired central regulation of energy homeostasis. Typically, high-fat feeding is characterised by an initial increase in energy consumption. A homeostatic response is then initiated that restores energy intake to pretreatment or nearly pretreatment levels [18]. Our lean, leptin-induced leptin-resistant rats apparently lack this homeostatic response, and as a result, following high-fat feeding, the energy consumption is maintained at an elevated level above that of the control high-fat-fed animals. This inability to downregulate energy consumption is probably a major contributor to the greater weight gain and adiposity in the lean, leptin-resistant rats fed the high-fat diet.

Glucose tolerance assessed 69 days after rAAV-leptin gene delivery revealed enhanced insulin sensitivity in rAAVleptin compared with control rats. Despite similar food intake in the two groups, the rAAV-leptin rats weighed less and had lower mean serum leptin and insulin levels, implying that these animals had less fat mass relative to the control animals. The insulin hypersensitivity may be a direct result of this reduction in adiposity. Any insulin hypersensitivity would favour fat deposition when excess substrate is available, hence contributing to the augmented adiposity following high-fat feeding in lean, leptin-resistant rats.

In summary, the present study indicates that elevated central leptin reduces maximal leptin signalling capacity and precipitates weight gain and adiposity following highfat feeding. The leptin-induced leptin resistance impairs the normal energy homeostasis following a high-fat diet, resulting in a persistent elevation in energy consumption. This creates a milieu that favours fat deposition and excess 
weight gain. We contend that leptin resistance is not only a consequence of obesity, but also one cause of obesity.

Acknowledgements This work was supported by the Medical Research Service of the Department of Veterans Affairs, USA, and a National Institute on Aging Grant (AG-17047).

\section{References}

1. Frederich RC, Hamann A, Anderson S, Lollmann B, Lowell BB, Flier JS (1995) Leptin levels reflect body lipid content in mice: evidence for diet-induced resistance to leptin action. Nat Med $1: 1311-1314$

2. Considine RV, Sinha MK, Heiman ML et al (1996) Serum immunoreactive leptin concentrations in normal-weight and obese humans. N Engl J Med 334:292-295

3. Halaas JL, Boozer C, Blair-West J, Fidahusein N, Denton DA, Friedman JM (1997) Physiological response to long-term peripheral and central leptin infusion in lean and obese mice. Proc Natl Acad Sci U S A 94:8878-8883

4. Widdowson PS, Upton R, Buckingham R, Arch J, Williams G (1997) Inhibition of food response to intracerebroventricular injection of leptin is attenuated in rats with diet-induced obesity. Diabetes 46:1782-1785

5. Caro JF, Kolaczynski JW, Nyce MR et al (1996) Decreased cerebrospinal-fluid/serum leptin ratio in obesity: a possible mechanism for leptin resistance. Lancet 348:159-161

6. Scarpace PJ, Matheny M, Pollock BH, Tumer N (1997) Leptin increases uncoupling protein expression and energy expenditure. Am J Physiol 273:E226-E230

7. Elmquist JK, Maratos-Flier E, Saper CB, Flier JS (1998) Unraveling the central nervous system pathways underlying responses to leptin. Nat Neurosci 1:445-450

8. Friedman JM, Halaas JL (1998) Leptin and the regulation of body weight in mammals. Nature 395:763-770

9. Scarpace PJ, Matheny M, Zhang Y et al (2002) Central leptin gene delivery evokes persistent leptin signal transduction in young and aged-obese rats but physiological responses become attenuated over time in aged-obese rats. Neuropharmacology 42:548-561

10. Scarpace PJ, Matheny M, Zolotukhin S, Tumer N, Zhang Y (2003) Leptin-induced leptin resistant rats exhibit enhanced responses to the melanocortin agonist MT II. Neuropharmacology 45:211-219

11. Scarpace PJ, Matheny M, Zhang Y et al (2002) Leptin-induced leptin resistance reveals separate roles for the anorexic and thermogenic responses in weight maintenance. Endocrinology 143:3026-3035
12. Wilsey J, Scarpace PJ (2004) Caloric restriction reverses the deficits in leptin receptor protein and leptin signaling capacity associated with diet-induced obesity: role of leptin in the regulation of hypothalamic long-form leptin receptor expression. J Endocrinol 181:297-306

13. Chen G, Koyama K, Yuan X et al (1996) Disappearance of body fat in normal rats induced by adenovirus-mediated leptin gene therapy. Proc Natl Acad Sci U S A 93:14795-14799

14. Zolotukhin S, Byrne BJ, Mason E et al (1999) Recombinant adeno-associated virus purification using novel methods improves infectious titer and yield. Gene Ther 6:973-985

15. Scarpace PJ, Matheny M, Tumer N (2001) Hypothalamic leptin resistance is associated with impaired leptin signal transduction in aged obese rats. Neuroscience 104:1111-1117

16. Ahima RS, Flier JS (2000) Leptin. Annu Rev Physiol 62:413437

17. Van Heek M, Compton DS, France CF et al (1997) Diet-induced obese mice develop peripheral, but not central, resistance to leptin. J Clin Invest 99:385-390

18. Wilsey J, Zolotukhin S, Prima V, Scarpace PJ (2003) Central leptin gene therapy fails to overcome leptin resistance associated with diet-induced obesity. Am J Physiol Regul Integr Comp Physiol 285:R1011-R1020

19. Bjorbaek C, El-Haschimi K, Frantz JD, Flier JS (1999) The role of SOCS3 in leptin signaling and leptin resistance. J Biol Chem 274:30059-30065

20. Pal R, Sahu A (2003) Leptin signaling in the hypothalamus during chronic central leptin infusion. Endocrinology 144:37893798

21. Martin RL, Perez E, He YJ, Dawson R Jr, Millard WJ (2000) Leptin resistance is associated with hypothalamic leptin receptor mRNA and protein downregulation. Metabolism 49:1479-1484

22. Wilsey J, Zolotukhin S, Prima V, Shek EW, Matheny MM, Scarpace PJ (2002) Hypothalamic delivery of doxycycline-inducible leptin gene allows for reversible transgene expression and physiological responses. Gene Ther 9:1492-1499

23. Niswender KD, Morrison CD, Clegg DJ et al (2003) Insulin activation of phosphatidylinositol 3-kinase in the hypothalamic arcuate nucleus: a key mediator of insulin-induced anorexia. Diabetes 52:227-231

24. Niswender KD, Morton GJ, Stearns WH, Rhodes CJ, Myers MG Jr, Schwartz MW (2001) Intracellular signalling. Key enzyme in leptin-induced anorexia. Nature 413:794-795

25. Huang W, Dedousis N, Bhatt BA, O'Doherty RM (2004) Impaired activation of phosphatidylinositol 3-kinase by leptin is a novel mechanism of hepatic leptin resistance in diet-induced obesity. J Biol Chem 279:21695-21700

26. Ogus S, Ke Y, Qiu J, Wang B, Chehab FF (2003) Hyperleptinemia precipitates diet-induced obesity in transgenic mice overexpressing leptin. Endocrinology 144:2865-2869 